\title{
Comment on "Ideal Capacitor Circuits and Energy Conservation" by K. Mita and M. Boufaida [Am. J. Phys. 67 (8), 737-739 (1999)]
}

\author{
Asim Gangopadhyaya \\ Loyola University Chicago, agangop@luc.edu \\ Jeffrey Mallow \\ Loyola University Chicago, jmallow@luc.edu
}

Follow this and additional works at: https://ecommons.luc.edu/physics_facpubs

Part of the Other Physics Commons, and the Quantum Physics Commons

\section{Recommended Citation}

Gangopadhyaya, A. and Mallow, J. (1993). Comment on "ideal capacitor circuits and energy conservation" by K. Mita and M. Boufaida [Am. J. Phys. 67 (8), 737-739 (1999)]. American Journal of Physics, 68 , 670-672.

This Article is brought to you for free and open access by the Faculty Publications and Other Works by Department at Loyola eCommons. It has been accepted for inclusion in Physics: Faculty Publications and Other Works by an authorized administrator of Loyola eCommons. For more information, please contact ecommons@luc.edu. \section{(c) (i) $\Theta$}

This work is licensed under a Creative Commons Attribution-Noncommercial-No Derivative Works 3.0 License. (c) American Association of Physics Teachers, 1999. 


\title{
Uniqueness of the angular velocity of a rigid body: Correction of two faulty proofs
}

\author{
Nivaldo A. Lemos ${ }^{\text {a) }}$ \\ Instituto de Física, Universidade Federal Fluminense, Av. Litorânea s/n, 24210-340 Boa Viagem, Niterói, \\ RJ, Brazil
}

(Received 25 June 1999; accepted 3 August 1999)

The angular velocity is an absolute or intrinsic property of a rigid body; that is, all points of a rotating rigid body have the same angular velocity. This fact is well known, but its proof is often erroneous. Here we correct two faulty proofs of this result, one in Goldstein's famous textbook and the other published nearly 30 years ago in this journal. (C) 2000 American Association of Physics Teachers.

All points of a rotating rigid body have the same angular velocity or, equivalently, the instantaneous angular velocity vector does not depend on the choice of the origin of the system of axes attached to the body. Although this is intuitively clear, a rigorous proof is necessary.

Goldstein's proof runs as follows. ${ }^{1}$ Let $\Sigma_{1}$ and $\Sigma_{2}$ be two coordinate systems attached to the body, and let $\mathbf{R}_{1}$ and $\mathbf{R}_{2}$ be the position vectors of their corresponding origins $O_{1}$ and $\mathrm{O}_{2}$ with respect to the origin $\mathrm{O}$ of an inertial reference frame $\Sigma$, as depicted in Fig. 1. Assume the angular velocities pertaining to $\Sigma_{1}$ and $\Sigma_{2}$ are $\boldsymbol{\omega}_{1}$ and $\boldsymbol{\omega}_{2}$, respectively.

Setting $\mathbf{R}=\mathbf{R}_{2}-\mathbf{R}_{1}$ and regarding $O_{2}$ as a point defined relative to $\Sigma_{1}$, we can write the time derivative of $\mathbf{R}_{2}$ with respect to the inertial reference frame $\Sigma$ as

$$
\frac{d \mathbf{R}_{2}}{d t}=\frac{d \mathbf{R}_{1}}{d t}+\frac{d \mathbf{R}}{d t}=\frac{d \mathbf{R}_{1}}{d t}+\boldsymbol{\omega}_{1} \times \mathbf{R},
$$

since $\mathbf{R}$ is a constant vector with respect to $\Sigma_{1}$. Similarly, considering $O_{1}$ as a point defined relative to $\Sigma_{2}$, we can write

$$
\frac{d \mathbf{R}_{1}}{d t}=\frac{d \mathbf{R}_{2}}{d t}-\frac{d \mathbf{R}}{d t}=\frac{d \mathbf{R}_{2}}{d t}-\boldsymbol{\omega}_{2} \times \mathbf{R} .
$$

Now Goldstein states that a comparison of Eqs. (1) and (2) shows that $\boldsymbol{\omega}_{1}=\boldsymbol{\omega}_{2}$. As a matter of fact, from (1) and (2) all one can deduce is

$$
\left(\boldsymbol{\omega}_{1}-\boldsymbol{\omega}_{2}\right) \times \mathbf{R}=0 .
$$

Goldstein seems to imply that the equality of the two angular velocities follows from the arbitrariness of $\mathbf{R}$, although he does not explicitly say so. However, this argument is incorrect for a subtle reason. By hypothesis, any changes in $\mathbf{R}$ induce changes in either $\boldsymbol{\omega}_{1}$ or $\boldsymbol{\omega}_{2}$, or in both. Therefore, the equality of $\boldsymbol{\omega}_{1}$ and $\boldsymbol{\omega}_{2}$ cannot be inferred from Eq. (3).

A slightly different line of reasoning is undertaken by Gruber. ${ }^{2}$ Let $P$ be an arbitrary point of the rigid body with position vectors $\mathbf{r}_{1}$ and $\mathbf{r}_{2}$ with respect to the origins $O_{1}$ and $\mathrm{O}_{2}$, respectively. From Fig. 1 one reads $\mathbf{r}=\mathbf{R}_{1}+\mathbf{r}_{1}=\mathbf{R}_{2}$ $+\mathbf{r}_{2}$, so that

$$
\frac{d \mathbf{r}}{d t}=\frac{d \mathbf{R}_{1}}{d t}+\frac{d \mathbf{r}_{1}}{d t}=\frac{d \mathbf{R}_{1}}{d t}+\omega_{1} \times \mathbf{r}_{1}
$$

and, similarly,

$$
\frac{d \mathbf{r}}{d t}=\frac{d \mathbf{R}_{2}}{d t}+\boldsymbol{\omega}_{2} \times \mathbf{r}_{2},
$$

because $\mathbf{r}_{1}$ and $\mathbf{r}_{2}$ are constant vectors from the point of view of every coordinate system attached to the body. After some algebraic manipulations, Gruber is led to

$$
\left(\boldsymbol{\omega}_{2}-\boldsymbol{\omega}_{1}\right) \cdot\left(\mathbf{r}_{2} \times \mathbf{r}_{1}\right)=0
$$

Then he argues that $\mathbf{r}_{2} \times \mathbf{r}_{1}$ is arbitrary as a consequence of the arbitrariness of $\mathbf{r}_{1}$ and $\mathbf{r}_{2}$; hence there ensues the equality of $\boldsymbol{\omega}_{1}$ and $\boldsymbol{\omega}_{2}$. This argument is flawed, however, since the vector $\mathbf{r}_{2} \times \mathbf{r}_{1}$ always lies on a plane perpendicular to $\mathbf{R}$, so that Eq. (6) allows one only to conclude that $\boldsymbol{\omega}_{1}$ and $\boldsymbol{\omega}_{2}$ differ by a vector proportional to $\mathbf{R}$.

Gruber's reasoning may be made rigorous as follows. Writing $\mathbf{R}=\mathbf{R}_{2}-\mathbf{R}_{1}=\mathbf{r}_{1}-\mathbf{r}_{2}$, from (4) and (5) one finds

$$
\frac{d \mathbf{R}}{d t}=\boldsymbol{\omega}_{1} \times \mathbf{r}_{1}-\boldsymbol{\omega}_{2} \times \mathbf{r}_{2} .
$$

On the other hand,

$$
\frac{d \mathbf{R}}{d t}=\boldsymbol{\omega}_{1} \times \mathbf{R}
$$

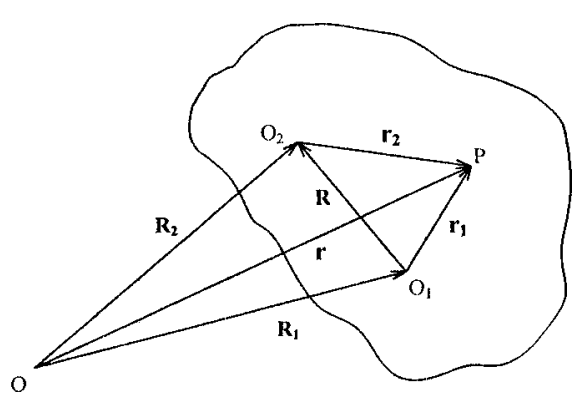

Fig. 1. $O_{1}$ and $O_{2}$ are the origins of two coordinate systems attached to a rotating rigid body. 
because $\mathbf{R}$ is also a constant vector with respect to the coordinate system $\Sigma_{1}$ fixed on the body. Combining (7) and (8) we obtain

$$
\boldsymbol{\omega}_{1} \times \mathbf{R}=\boldsymbol{\omega}_{1} \times \mathbf{r}_{1}-\boldsymbol{\omega}_{2} \times \mathbf{r}_{2}
$$

or, inasmuch as $\mathbf{R}=\mathbf{r}_{1}-\mathbf{r}_{2}$,

$$
\left(\boldsymbol{\omega}_{2}-\boldsymbol{\omega}_{1}\right) \times \mathbf{r}_{2}=0 .
$$

Since $P$ is any point of the rigid body, $\mathbf{r}_{2}$ is an arbitrary vector that can be freely changed without affecting either $\boldsymbol{\omega}_{1}$ or $\boldsymbol{\omega}_{2}$. As an immediate consequence $\boldsymbol{\omega}_{2}=\boldsymbol{\omega}_{1}$, and the proof is complete.

${ }^{a)}$ Electronic mail: nivaldo@if.uff.br

${ }^{1}$ H. Goldstein, Classical Mechanics (Addison-Wesley, Reading, MA, 1980), 2nd ed., pp. 189-190.

${ }^{2}$ G. R. Gruber, "Clarification of two important questions in rigid body mechanics," Am. J. Phys. 40, 421-423 (1972).

\title{
Fourier transform solution to the semi-infinite resistance ladder
}

\author{
R. M. Dimeo \\ National Institute of Standards and Technology, 100 Bureau Drive-Stop 8562, Gaithersburg, \\ Maryland 20899-8562
}

(Received 16 August 1999; accepted 20 September 1999)

In a recent $\operatorname{article}^{1}$ a general method for solving for the resistance between any two nodes of a number of infinite resistance lattices using discrete-variable Fourier transforms ${ }^{2}$ was presented. In this note the same technique is applied to the semi-infinite ladder network. The mathematical methods used in the solution to this problem (Fourier transforms and contour integration) are well within the abilities of the undergraduate physics major. This one-dimensional example is thus complementary to the two (and higher)-dimensional networks presented in the previous article, ${ }^{1}$ and this example can be used in a junior-level mathematical methods course.

The input resistance of the semi-infinite ladder network composed of identical resistors, shown in Fig. 1, is well known and can be solved by using simple rules of parallel and series combinations of resistors (see the paper by Srinivasan $^{3}$ and references therein). One simply notes that adding on another resistive repeat unit to the semi-infinite ladder will not affect the overall input resistance so that $R_{\text {eq }}=R_{\text {eq }}^{\prime}$. The equivalent resistance is thus found to be equal to the golden ratio multiplied by the unit of resistance, $R_{\text {eq }}$ $=[(1+\sqrt{5}) / 2] R$.

This result can be found using the Fourier transform solution to the difference equation governing the auxiliary resistance ladder shown in Fig. 2(a). This auxiliary ladder, which we introduce for mathematical convenience, is infinite in

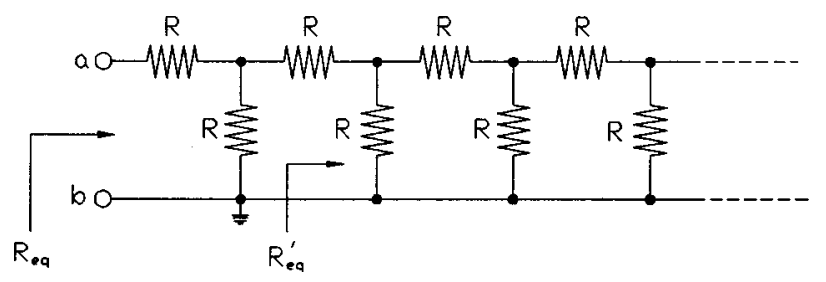

Fig. 1. The semi-infinite resistor ladder. The resistance between nodes a and $\mathrm{b}$ is defined as the input resistance for this network. both directions, whereas the ladder of primary interest is semi-infinite (infinite in only one direction). We can easily relate the currents and voltages of the infinite ladder to those of the semi-infinite ladder. If we know that a node voltage, $v_{0}$, results from the input of 1 A of current at the same node, then the resistance, $R_{\text {eq }}$, can be found, as shown in Fig. 2(b). Simple application of the current rule to Fig. 2(b) yields

$$
1=\frac{v_{0}}{R}+\frac{2 v_{0}}{R_{\mathrm{eq}}} \Rightarrow R_{\mathrm{eq}}=\frac{2 v_{0} R}{R-v_{0}} .
$$

We can determine the node voltage at any node, $n$, based on the current entering that node in the infinite ladder of Fig.

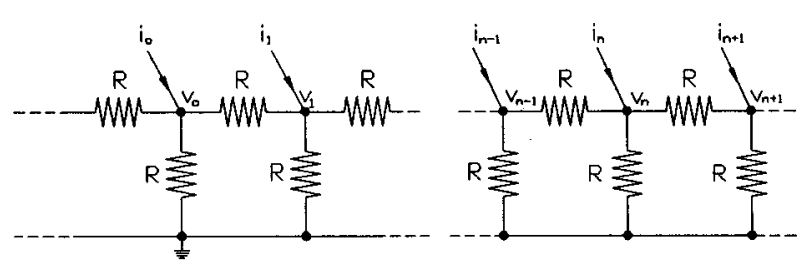

(a)

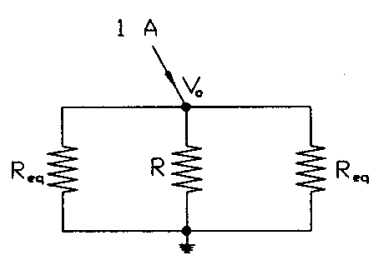

(b).

Fig. 2. Reduction of (a) the infinite resistor ladder to (b) a circuit in terms of the equivalent resistance between nodes $\mathrm{a}$ and $\mathrm{b}$ of the semi-infinite ladder shown in Fig. 1. Note that all of the external currents being fed into the network in (a) are set to zero except $i_{0}=1 \mathrm{~A}$ for the specific case of making the equivalence to the circuit shown in Fig. 1. 
2(a). Kirchhoff's current law applied to node $n$ results in the following difference equation,

$$
R i_{n}=3 v_{n}-v_{n+1}-v_{n-1},
$$

where $i_{n}$ is current fed into node $n$ supplied by an external source.

Following the method presented in Atkinson's paper, ${ }^{1}$ we use the discrete-variable Fourier transform pair (also called a complex Fourier series),

$$
x_{n}=\frac{1}{2 \pi} \int_{0}^{2 \pi} d \beta X(\beta) e^{i n \beta} \leftrightarrow X(\beta)=\sum_{n=-\infty}^{\infty} x_{n} e^{-i n \beta},
$$

and transform the difference equation from $n$ to $\beta$. The resulting transformed voltage is

$$
v(\beta)=\frac{R I(\beta)}{3-2 \cos \beta},
$$

with the actual voltage at node $n$ expressed as the integral

$$
v_{n}=\frac{R}{2 \pi} \int_{0}^{2 \pi} d \beta \frac{I(\beta)}{3-2 \cos \beta} e^{i n \beta} .
$$

A current of $1 \mathrm{~A}$ fed into node 0 as shown in Fig. 2, represented as $i_{n}=\delta_{n, 0}$ (where $\delta_{n, k}=1$ if $n=k$ and 0 otherwise), results in a transformed current of $I(\beta)=1$. Therefore, the voltage at any node, $n$, under these circumstances is written as

$$
v_{n}=\frac{R}{2 \pi} \int_{0}^{2 \pi} d \beta \frac{e^{i n \beta}}{3-2 \cos \beta}
$$

The voltage at node $0, v_{0}$, can be evaluated readily using contour integration. The integral which we wish to solve is

$$
v_{0}=\frac{R}{2 \pi} \int_{0}^{2 \pi} \frac{d \beta}{3-2 \cos \beta}
$$

with the corresponding contour integral

$$
v_{0}=\frac{R}{2 \pi i} \oint_{|z|=1} \frac{d z}{z} \frac{1}{3-\left(z+z^{-1}\right)} \text {. }
$$

The integrand has only one pole located in the unit circle, $z=(3-\sqrt{5}) / 2$. Therefore, the integral evaluates to $v_{0}$ $=R / \sqrt{5}$, which is the effective resistance of the infinite ladder. Finally, we obtain the effective resistance of the semiinfinite ladder by substitution of $v_{0}$ into Eq. (1) and obtain $R_{\text {eq }}=[(1+\sqrt{5}) / 2] R$.

${ }^{1}$ D. Atkinson and F. J. van Steenwijk, "Infinite resistive lattices,'” Am. J. Phys. 67, 486-492 (1999).

${ }^{2}$ A. V. Oppenheim and R. W. Schafer, Discrete-Time Signal Processing (Prentice-Hall, Englewood Cliffs, NJ, 1989).

${ }^{3}$ T. P. Srinivasan, "Fibonacci sequence, golden ratio, and a network of resistors,'” Am. J. Phys. 60, 461-462 (1992).

\title{
Comment on "Ideal capacitor circuits and energy conservation," by K. Mita and M. Boufaida [Am. J. Phys. 67 (8), 737-739 (1999)]
}

\author{
A. Gangopadhyaya and J. V. Mallow \\ Department of Physics, Loyola University Chicago, Chicago, Illinois 60626
}

(Received 19 August 1999; accepted 27 October 1999)

K. Mita and M. Boufaida (hereinafter MB) ${ }^{1}$ discuss the puzzle of the missing energy in a capacitor charged from a power supply (a battery or another capacitor), with neither resistance nor inductance in the circuit. In such a circuit, the power supply appears to deliver energy $E_{\mathrm{PS}}=q V_{0}$, while the capacitor only stores $\frac{1}{2} C V_{0}^{2}=\frac{1}{2} q V_{0}$. The problem disappears if either inductance $L$ or resistance $R$ is introduced into the circuit, where $L$ and $R$ can be as small as one likes, but not zero (a rather peculiar discontinuity). MB note that the function of $L$ and/or $R$ is to change a discontinuous, instantaneous charging process into a continuous one, with a finite time constant. They then generalize their observation, and show that any power supply described by $V=V_{0} f(t)$, where $f(t)$ is a monotonically increasing, continuous, differentiable function of time, will deliver the "correct" energy. With $0<t<t_{0}, f(0)=0, f\left(t_{0}\right)=1$, and $q=C V$,

$$
\begin{aligned}
E_{\mathrm{PS}}=\int \frac{d q}{d t} V d t=C V_{0}^{2} \int f(t) \frac{d f}{d t} d t & =C V_{0}^{2} \int f d f \\
& =\frac{1}{2} C V_{0}^{2} .
\end{aligned}
$$

MB note that the charge across the instantaneously charging capacitor can be written as $q=C V_{0} \Theta(t)$, where $\Theta(t)$ is the Heaviside step-function: 


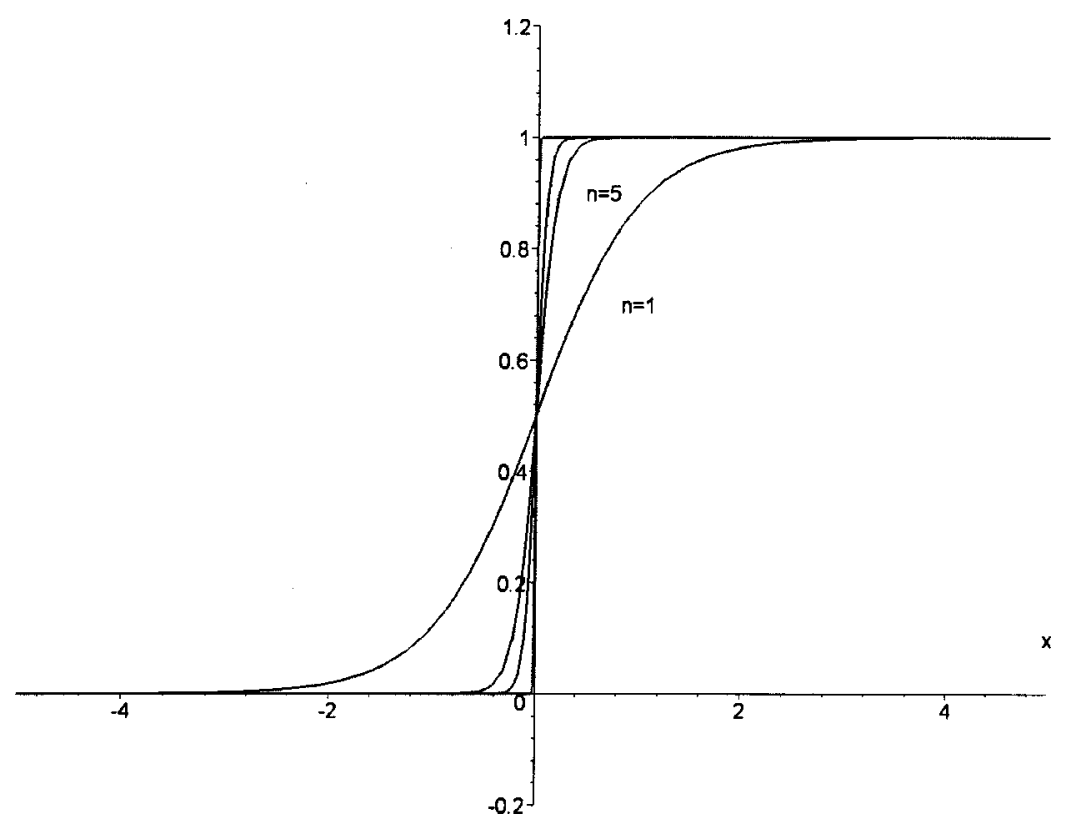

Fig. 1. Function $f(x)=\frac{1}{2}[1+\tanh (n x)]$ is plotted for $n$ $=1,5,10,100$.

$$
\Theta(t)=\left\{\begin{array}{ll}
1, & t>0 \\
0, & t<0
\end{array} .\right.
$$

They point out that $\Theta(t)$ is not continuous, and therefore fails to meet one of the validity criteria for Eq. (1). They attribute the missing energy puzzle to this feature. They go on to observe that $\Theta(t)$ can be written as

$$
\Theta(t)=\lim _{n \rightarrow \infty} \frac{1}{2}[1+\tanh (n t)] .
$$

The function $\frac{1}{2}[1+\tanh (n t)]$ is continuous for $n<\infty$, and has the value $\frac{1}{2}$ at $t=0$. MB note that using this in Eq. (1) would immediately give $E_{\mathrm{PS}}=\frac{1}{2} C V_{0}^{2}$, and the conundrum would be resolved. However, they express reservations as to the appropriateness or the meaning of passing to the $n \rightarrow \infty$ limit.

We believe that MB's formulation in Eq. (1) is sufficiently robust as to be applicable to $\Theta(t)$ itself, despite its discontinuity. To do this requires some reexamination of the limits of integration of Eq. (1), as well as the limiting procedure in Eq. (2).

Let us examine the properties of the function $f(t)$ $=\frac{1}{2}[1+\tanh (n t)]$, shown in Fig. 1, for various $n$. Clearly, as MB note, for all finite $n, f(0)=\frac{1}{2}$. But what about infinite $n$ ? If we calculate the integral as in Eq. (1), we obtain

$$
\int f(t) \frac{d f}{d t} d t=\int f d f=\frac{1}{2} f^{2}=\frac{1}{8}[1+\tanh (n t)]^{2} .
$$

What should be our limits of integration? MB take $f(0)=0$ and $f\left(t_{0}\right)=1$, where $t_{0}$ can be $\infty$. The motivation for MB's formulation of Eq. (1) is to examine energy conservation. Consistent with that formulation, then, in order to give the same "area under the curve" as MB's functions $f(t)$, the limits of integration for $\frac{1}{2}[1+\tanh (n t)]$ must be taken as $t= \pm \infty$. $\operatorname{Tanh}(-\infty)=-1, \tanh (\infty)=1$. The integral is thus equal to $\frac{1}{2}$, independent of the value of $n$. Therefore, the numerical value of Eq. (3) is $\frac{1}{2}$ for all $n$, including $\infty$, and the results of Eq. (1) are valid for the Heaviside step function $\Theta(t)$ as defined in Eq. (2). The only apparent effect of taking $n \rightarrow \infty$ is to make $f(-\infty)=f\left(0_{-}\right)$, the original lower limit of integration in Eq. (1).

Does this resolve the missing energy problem? Yes and no. The limiting procedure is equivalent to placing a resistor in the circuit, then letting its value go to zero. In particular, $\frac{1}{2}[1+\tanh (n t)]=e^{n t} /\left[e^{n t}+e^{-n t}\right]$, which has the same behavior for $n \rightarrow \infty$ as does $\left[1-e^{-t / R C}\right]$ for $R \rightarrow 0$. The peculiarity noted above, that $R$ (or $L$ ) can be as close to zero as one likes, but not exactly zero, then disappears: $E_{\mathrm{PS}}=\frac{1}{2} C V_{0}^{2}$ both for nonzero and zero resistance (or inductance). On the other hand, the battery now appears to be delivering $\frac{1}{2} q V_{0}$, half of what we normally expect. In other words, the puzzle seems to have simply shifted from "Where'd the missing energy go?" to "What's wrong with the battery?"' One could argue that this is where the puzzle belongs. The ideal battery without internal resistance produces infinite current over zero time. If we write the voltage difference across a real battery as $V_{0}=\mathcal{E}-i r$, where $\mathcal{E}$ is the emf and $r$ is the internal resistance of the battery, then letting $r \rightarrow 0$ while $i \rightarrow \infty$ gives an indeterminate $V_{0}$. What then can it mean to say that "the ideal battery does work $q V_{0}$ "'? Why not $\frac{1}{2} q V_{0}$ ? Or zero? Or some other value?

Introducing resistance, inductance, or indeed any wellbehaved time-dependent $V$, as shown by $\mathrm{MB}$, performs precisely the function of making the scenario realistic, by requiring a well-defined closed-circuit battery voltage. Concomitantly, this yields a finite current $i=d q / d t$. But, as our calculation suggests, these stratagems may not be necessary, as long as we do not insist that we know that the ideal battery is delivering $q V_{0}$. Rather than hunting for missing energy, it is just as reasonable to interpret our result as demonstrating that an ideal battery delivers only $\frac{1}{2} q V_{0}$.

Finally, we note that the same argument holds, as it must, for the mechanical analogue of stretching a massless spring of force constant $k$ a total distance $d$ with no viscosity, as discussed by MB. A constant force $F_{0}$, set equal to $k d$, 
stretching the spring, appears to do work equal to $k d^{2}$, while the spring only stores $\frac{1}{2} k d^{2}$. But what does such a force, acting neither on a mass nor against a viscous medium, actually deliver? Neither velocity nor acceleration can be determined; thus, any claims about the numerical values of work and energy are suspect. Add in a mass and/or a velocity-dependent dissipative term, and the problem disappears.

\section{ACKNOWLEDGMENT}

We would like to thank Professor Katsunori Mita for important discussion on this topic and also for bringing a relevant article to our attention. ${ }^{2}$

${ }^{1}$ K. Mita and M. Boufaida, Am. J. Phys. 67 (8), 737-739 (1999). ${ }^{2}$ D. Griffiths and S. Walborn, "Dirac deltas and discontinuous functions," Am. J. Phys. 67 (5), 446-447 (1999).

\title{
Comment on "A quantum bouncing ball," by Julio Gea-Banacloche [Am. J. Phys. 67 (9), 776-782 (1999)]
}

\author{
Olivier Vallée ${ }^{\text {a) }}$ \\ LASEP, Centre Universitaire de Bourges, rue Gaston Berger BP 4043, 18028, Bourges Cedex, France
}

(Received 18 October 1999; accepted 3 January 2000)

In a recent paper, J. Gea-Banacloche ${ }^{1}$ investigated the quantum analog of the classical bouncing ball. It was certainly a big task of computing to create this nice movie (http: //www.uark.edu/misc/julio/bouncing_ball/bouncing_ ball.html). The aim of these comments is to point out that things might be a little simpler with analytical integrals.

(a) Primitive of the square of Airy functions. There are at least three references (Refs. 2-4) that give the primitive corresponding to the formula (13) of the author:

$$
\int_{0}^{\infty} \operatorname{Ai}^{2}\left(z-z_{n}\right) d z=u \mathrm{Ai}^{2}(u)-\left.\mathrm{Ai}^{\prime 2}(u)\right|_{-z_{n}} ^{\infty}=\operatorname{Ai}^{\prime 2}\left(-z_{n}\right)
$$

As a consequence, a parity phase factor could as well be included in the normalization coefficient, which then reads:

$$
\mathcal{N}_{n}^{*}=\mathrm{Ai}^{\prime}\left(-z_{n}\right)=(-1)^{n-1}\left|\mathrm{Ai}^{\prime}\left(-z_{n}\right)\right|,
$$

as is usually done for instance with the hydrogen atom, ${ }^{5}$ in place of the author's $\mathcal{N}_{n}=\left|\mathrm{Ai}^{\prime}\left(-z_{n}\right)\right|$. But this is unimportant for the purpose of the paper, for it is a pure matter of convention.

(b) The dipole matrix elements. The matrix elements $\langle m|z| n\rangle$ can also be found in the literature: ${ }^{3}$

$$
\begin{aligned}
\int_{0}^{\infty} & \operatorname{Ai}\left(z-z_{n}\right) \operatorname{Ai}\left(z-z_{m}\right) z d z \\
& =\frac{-2}{\left(z_{n}-z_{m}\right)^{2}} \operatorname{Ai}^{\prime}\left(-z_{n}\right) \mathrm{Ai}^{\prime}\left(-z_{m}\right) .
\end{aligned}
$$

That is to say: $\langle m|z| n\rangle=2(-1)^{n+m-1} /\left(z_{n}-z_{m}\right)^{2}$, with the normalization constant $\mathcal{N}_{n}$ of the author. The diagonal elements are also found from ${ }^{3}$

$$
\langle n|z| n\rangle=\mathcal{N}_{n}^{2} \int_{0}^{\infty} \operatorname{Ai}^{2}\left(z-z_{n}\right) z d z=\frac{2}{3} z_{n}
$$

A result that was numerically verified by the author. Actually, all these integrals are available on the web at: http://integrals.wolfram.com/index.cgi. ${ }^{6}$

(c) The $C_{n}$ coefficients in the eigenfunction expansion. For a Gaussian initial wave packet, the hypothesis $\sigma \ll z_{0}$ (assumed by the author) means that this packet is sharply peaked at $z_{0}$, so a simple approximation of this packet is a Dirac delta $\delta\left(z-z_{0}\right)$, but this is perhaps too crude an approximation. Another way is to extend, like the author, the lower bound of the integral Eq. (16) to $-\infty$. In this case the result is analytic: ${ }^{4}$

$$
\begin{aligned}
C_{n} \simeq & \mathcal{N}_{n}\left(\frac{2}{\pi \sigma^{2}}\right)^{1 / 4} \int_{-\infty}^{\infty} \operatorname{Ai}\left(z-z_{n}\right) e^{-\left(z-z_{0}\right)^{2} / \sigma^{2}} d z \\
= & \mathcal{N}_{n}\left(\frac{2}{\pi \sigma^{2}}\right)^{1 / 4} \sqrt{\pi} \sigma \exp \left[\frac{\sigma^{2}}{4}\left(z_{0}-z_{n}+\frac{\sigma^{4}}{24}\right)\right] \\
& \times \operatorname{Ai}\left(z_{0}-z_{n}+\frac{\sigma^{4}}{16}\right) .
\end{aligned}
$$

Despite these small (calculus) details, the main conclusions of this paper are very interesting for they outline the fundamental differences between classical and quantum mechanics. In particular the maxima of probability do not reflect the usual viewpoint of classical mechanics: The particle does not "spend" so much time at these maxima.

\footnotetext{
${ }^{a)}$ Electronic mail: Olivier.Vallee@univ-orleans.fr

${ }^{1}$ J. Gea-Banacloche, "A quantum bouncing ball," Am. J. Phys. 67, 776782 (1999).

${ }^{2}$ J. R. Albright, "Integrals of products of Airy functions," J. Phys. A 10, 485-490 (1977).

${ }^{3}$ R. G. Gordon, "New method for constructing wave functions for bound states and scattering," J. Chem. Phys. 51, 14-25 (1969).
} 
${ }^{4}$ O. Vallée and M. Soares, Les Fonctions d'Airy Pour la Physique, ISBN: 2-84352-090-8 (Diderot Ed., 1998).

${ }^{5} \mathrm{H}$. Bethe and E. Salpeter, Quantum Mechanics of One- and Two-electron Atoms (Springer-Verlag, Berlin, 1957).
${ }^{6}$ The syntax of Airy functions in Mathematica is AiryAi[x] for Ai(x) and AiryAiPrime[x] for its derivative. The "Integrator" gives the primitive, but not the integral of a given function; then the result should be finished by hand.

\section{NAVAL CALCULUS}

When I was teaching mathematics to future naval officers during the war, I was told that the Navy had found that men who had studied calculus made better line officers than men who had not studied calculus. Nothing is clearer (it was clear even to the Navy) than that a line officer never has the slightest use for calculus. At the most, his duties may require him to look up some numbers in tables and do a little arithmetic with them, or possibly substitute them into formulas ... Now we can understand why calculus improves the line officer. He needs to practice very simple kinds of mathematics; he gets this practice in less distasteful form by studying more advanced mathematics.

Ralph P. Boas, Jr., "“If this be Treason...,', in Lion Hunting \& Other Mathematical Pursuits, edited by Gerald L. Alexanderson and Dale H. Mugler (Mathematical Association of America, Washington, 1995), p. 224. 